\title{
Post-Operative Infections Associated with Cataract Surgery
}

\author{
Baseer U. Ahmad ${ }^{1}$, Igor Estrovich ${ }^{1}$ and Thomas Steinemann ${ }^{2}$ \\ ${ }^{1}$ Department of Ophthalmology, Cole Eye Institute, \\ Cleveland Clinic Foundation, Cleveland, Ohio \\ ${ }^{2}$ Department of Ophthalmology, MetroHealth Medical Center, Cleveland, Ohio
}

USA

\section{Introduction}

Post-operative surgical infection represents an uncommon but potentially devastating complication of cataract surgery. In the past several decades, cataract surgery has made major advances with the introduction of small-incision phacoemulsification, viscoelastic substances, and improved lens designs, as well as refinement of surgical techniques. As a result, postoperative care has become simpler and visual recovery has become much faster. Due to these factors, the incidence of post-operative infectious complications has also generally declined. In fact, the incidence of post-operative endophthalmitis has decreased since the mid-1900s from $0.5 \%$ to $0.04 \%-0.41 \%$ in the early $21^{\text {st }}$ century (Kattan, et al, Ravindran, et al). In the past decade, however, these rates appear to have increased due to the greater use of clear corneal incision techniques. As such, it is fundamentally important for the cataract surgeon to be familiar with recognition, etiology, and management of post-operative endophthalmitis.

\section{Epidemiology}

By most accounts, the incidence of post-operative endophthalmitis is consistently less than 1 in 1000 cases. There appears to be little geographic variability in the incidence of acute postoperative endophthalmitis. When comparing the largest incidence studies of respective countries, the risk in developed countries such as the United States $(0.09 \%)$, Canada $(0.043 \%)$, and Great Britain $(0.099 \%)$ is comparable to the risk in less developed countries like India $(0.09 \%)$ and Greece $(0.08 \%)$ (Kalpadakis, et al, Kattan, et al, Lloyd\&Braga-Mele, Mollan, et al, Ravindran, et al).

\section{Clinical presentation}

Post-operative infections related to cataract surgery primarily include endophthalmitis and corneal suture infection. Endophthalmitis may present acutely or in a more chronic form, depending on the causative organism. Symptoms of endophthalmitis include decreased vision, mild to severe ocular pain, photophobia, and floaters. The characteristic finding in endophthalmitis is vitreous inflammation, but it is often accompanied by other signs including anterior chamber cell and flare, hypopyon, ciliary injection, and corneal edema. 
Chronic cases may be more indolent with smoldering anterior chamber reaction, vitritis, and no significant external inflammation or pain. If the source of infection is suture-related, focal corneal infiltrate, anterior chamber reaction, and hypopyon may be early signs of a suture abscess and may develop into endophthalmitis if not treated promptly adequately.

\section{Etiology}

Using techniques of molecular identification, it has been demonstrated that an organism isolated from the vitreous was genetically indistinguishable from an isolate recovered from the patient's eyelid, conjunctiva, or nose in over $80 \%$ of cases. Speaker et al found an association between the external bacterial flora and the bacteria isolated from vitrectomy specimens in $82 \%$ of the patients with postoperative endophthalmitis (Speaker, et al). Similarly, the Endophthalmitis Vitrectomy Study (EVS) group found that the intraocular isolates were indistinguishable from conjunctival and lid specimens in $68 \%$ of the bacterial postoperative endophthalmitis cases. These and other similar series have supported the hypothesis that most cases of post-surgical endophthalmitis are caused by introduction of microorganisms from the conjunctiva and eyelid during operative manipulation and during the perioperative period.

However, intraocular contamination appears to be relatively common after uneventful cataract surgery; the rate of culture positivity in anterior chamber aspirate ranges from $0-46 \%$ and has shown that bacteria routinely enter the anterior chamber during cataract surgery (Dickey, et al, Leong, et al, Sherwood, et al, Srinivasan, et al). They may be carried into the eye by irrigation or may adhere to the intraocular lens or instruments as they are inserted through the incision. It is thought that the host reaction can overcome and prevent a bacterial infection in its initial phases as evidenced by the discrepancy between the high incidence of aqueous humor contamination and and the low incidence of postoperative endophthalmitis. The integrity of the lens capsule may also play a role in the prevention of infection (Dickey, et al).

Another potential cause of post-operative infection is the chronic colonization or adherence of bacteria to biomaterial. This tendency of relatively easy adherence can lead the development of a biofilm on the surface of the intraocular lens. Vafidis et al conducted a study in which an intraocular lens was placed across the conjunctival flap and section for 5 seconds during cataract surgery, and this resulted in a bacterial contamination rate of $26 \%$, demonstrating the ability of bacteria (mainly S. epidermidis, $87 \%$ ) to adhere instantaneously to intraocular lenses (Vafidis, et al). Propionibacterium acnes has also been commonly reported to form biofilms in orthopedic settings upon hardware (Holmberg, et al), and this bears similarity to the white, film-like deposits on intraocular lens surfaces that have been described in chronic endophthalmitis cases.

A rare category of post-operative surgical infection associated with cataract surgery is represented by suture complications. These have been described as early as weeks and as late as years after cataract surgery. Such abscesses can occur if the knots are unburied, broken, exposed, or loosely placed. Such sutures can accumulate mucus and act as a wick allowing a route for bacteria to penetrate the eye. Of note, another rare etiology described by a small case series has been of scleral flap necrosis and infectious endophthalmitis after cataract surgery, in which the scleral tunnels may have acted as abscess cavities (Ormerod, et al).

From a microbial standpoint, acute cases (occurring $<6$ weeks) are most commonly caused by gram-positive bacteria, of which coagulase-negative Staphylococcus (CoNS) is most often encountered (Table 1) (Kodjikian, et al). Chronic post-operative endophthalmitis (occurring $>6$ weeks) is usually due to lower virulence microorganisms, particularly P. acnes (Table 2) 
(Al-Mezaine, et al, Haapala, et al). Suture-related infection may occur at any time, but the inoculating organisms generally resemble those in acute post-surgical cases and case reports have described Streptococcus pneumoniae, staphylococcus aureus, and staphylococcus epidermidis growth from positive cultures.

\begin{tabular}{|l|c|l|c|}
\hline \multicolumn{2}{|c|}{$\begin{array}{c}\text { Endophthalmitis-Vitrectomy Study } \\
(1995, \mathrm{n}=420)\end{array}$} & \multicolumn{1}{c|}{$\begin{array}{c}\text { Endophthalmitis National Survey, France } \\
(2009, \mathrm{n}=95)\end{array}$} \\
\hline No growth & $17.9 \%$ & \multicolumn{1}{c|}{ No growth } & $52.6 \%$ \\
\hline Equivocal growth & $12.9 \%$ & Not precisely identified & $3.1 \%$ \\
\hline Gram +ve, coagulase -ve & $46.9 \%$ & Coagulase -ve Staphylococcus & $27.4 \%$ \\
\hline Other Gram +ve & $15.5 \%$ & Staphylococcus aureus & $6.3 \%$ \\
\cline { 3 - 4 } & $4.7 \%$ & Streptococcus & $7.4 \%$ \\
\hline Gram -ve & $3.8 \%$ & Gram -ve & $3.2 \%$ \\
\hline Polymicrobials & &
\end{tabular}

Table 1. Etiology of Acute Endophthalmitis

\begin{tabular}{|l|c|l|c|}
\hline \multicolumn{2}{|c|}{ Haapala, et al (2005, $\mathrm{n}=8)$} & \multicolumn{2}{c|}{ Al-Mezaine, et al (2009, $\mathrm{n}=17)$} \\
\hline Propionibacterium acnes & $75 \%$ & Propionibacterium acnes & $41.2 \%$ \\
\hline S. epidermidis & $12.5 \%$ & Coagulase -ve Staphylococcus & $17.6 \%$ \\
\hline \multirow{3}{*}{ Other Gram -ve } & \multirow{2}{*}{$12.5 \%$} & Polymicrobial & $17.6 \%$ \\
\cline { 3 - 4 } & & Fungal infection & $17.6 \%$ \\
\cline { 3 - 4 } & & Other Gram -ve & $6 \%$ \\
\hline
\end{tabular}

Table 2. Etiology of delayed-onset, culture proven endophthalmitis

\section{Treatment}

The landmark study in current treatment of acute postcataract endophmitis was the Endophthalmitis Vitrectomy Study (EVS) , a multicenter prospective randomized study that compared the effectiveness of immediate vitrectomy to vitreous tap with injection of intravitreal anbiotics.

In the EVS, patients with clinical evidence of acute postoperative endophthalmitis were randomly assigned to emergent vitreous tap or vitrectomy, with both groups receiving injection of intravitreal antibiotics ( $0.4 \mathrm{mg}$ amikacin and $1.0 \mathrm{mg}$ vancomycin). Patients were also given subconjunctival injections of antibiotics ( $25 \mathrm{mg}$ vancomycin, $100 \mathrm{mg}$ ceftazidime) and steroid (6 $\mathrm{mg}$ dexamethasone phosphate), topical fortified antibiotics $(50 \mathrm{mg} / \mathrm{ml}$ vancomycin and $20 \mathrm{mg} / \mathrm{ml}$ amikacin) and steroid (prednisolone acetate), as well as oral steroids (prednisone $30 \mathrm{mg}$ bid $\times$ 5-10 days). Patients were also randomly assigned to receive systemic IV antibiotics ( 2 g ceftazidime IV Q8h and $7.5 \mathrm{mg} / \mathrm{kg}$ amikacin IV followed by 6 $\mathrm{mg} / \mathrm{kg} \mathrm{Q} 12 \mathrm{~h}$ ) or no systemic antibiotics. Intravitreal steroids were not used.

Results and conclusions of the EVS included:

i. On average, signs and symptoms occurred 6 days after surgery $(75 \%$ presented within 2 weeks of surgery).

ii. Positive cultures were obtained in $69 \%$, of which $94 \%$ were Gram positive bacteria.

iii. Intravenous antibiotics were of no benefit and do not improve final outcome.

iv. Immediate vitrectomy had significant benefits only when patients presented with light perception vision or worse. Otherwise, emergent treatment with tap and intravitreal anbiotics should be given if presenting vision is better than light perception. 
v. Roughly half of patients in both the tap and vitrectomy groups achieved final visual acuity of $20 / 40$ or better ( $52.3 \%$ versus $53.7 \%$ respectively).

As a result of the EVS, acute endophthalmitis with vision better than light perception is usually treated with an anterior chamber/vitreous tap and intravitreal injection of vancomycin for Gram positive coverage, and amikacin or ceftazidime for Gram negative coverage.

For chronic endophthalmitis, no clear treatment strategies have been established. Propionibacterium acnes, the major causative organism, often forms capsular plaques and therapy with intravitreal antibiotics alone is associated with very high rates of recurrence. Instead, pars-plana vitrecomy with capsulectomy appears to be the most effective strategy based on studies involving relative large numbers of patients with delayed-onset $P$. acnes endopthalmitis (Aldave, et al, Clark, et al). Fungal etiologies are much rarer, more difficult to manage, and often treated with varying success using intravitreal antifungals such as amphotericin or voriconazole.

For suture-related infections, topical fortified antibiotic therapy may be adequate if the presentation is mild and if treated early in the course when the infection is confined to the cornea and anterior chamber. Appropriate initial therapy may include vancomycin (25-50 $\mathrm{mg} / \mathrm{ml}$ ) for gram-positive organisms, in combination with ceftazidime $(50 \mathrm{mg} / \mathrm{ml})$ or tobramycin $(15 \mathrm{mg} / \mathrm{ml})$ as frequently as every hour depending on the severity of presentation. In any suspected case of endophthalmitis or if progression is noted, early intravitreal antibiotics should be administered.

\section{Recent trends and advice on prevention}

Interestingly, the large variability in institutional methods such as sterilization of surgical instruments and patient flow in surgical suites seems to minimally impact the rate of infection. For instance, at the Aravind Eye Hospital in India, surgical instruments are sterilized using a short-cycle method, the surgeon alternates between two operating table in the same operating room, and uses the same sterile gloves for multiple cases. These studies collectively suggest that increasing facility efficiency and optimizing patient flow does not come at the expense of outcomes.

Preoperatively, recognition of blepharitis or predisposing risk factors is important. Case reports have described possibly inadequately treated blepharitis and rosacea associated with cases of endophthalmitis despite the use of good surgical technique. It is therefore prudent to treat significant cases of blepharitis, rosacea, and related disorders prior to surgery.

During the immediate perioperative period, commonly employed methods to reduce the risk of endophthalmitis include placing povidone-iodine 5\% drops in the conjunctival sac as part of the preoperative preparation of the eye, using adhesive drapes to isolate the lashes and lid margins from the operative field, and maintaining appropriate intraoperative aseptic technique (Ciulla, et al, Ou\&Ta). The effectiveness of perioperative topical antibiotics has been controversial. Topical therapy for 3 days prior to surgery can reduce bacterial counts but has not been shown to reduce the incidence of infection. In 2006, a preliminary report from a large European prospective multicenter randomized clinical trial indicated that injection of $1 \mathrm{mg}$ cefuroxime into the anterior chamber at the conclusion of cataract surgery can reduce the incidence of endophthalmitis fivefold. The validity of generalizing these results to all cataract procedures awaits publication of the final report. The use of vancomycin in infusion fluid during phacoemulsification has been proposed but remains controversial (Ciulla, et al, Ou\&Ta). Given the difficulty of obtaining preservative-free antibiotics that are commercially 
available in doses appropriate for intracameral prophylaxis, surgeons need to weigh these results against the risk of dilutional errors or preservative toxicity.

Paradoxically, recent surgical advances such as the use of sutureless clear corneal incisions and sutureless surgery have shown an increase in rates of endophthalmitis over the past decade (Lundstrom, et al, Taban, et al). While this small incidence represents a modest risk for individuals undergoing cataract extraction, the global volume of the most common surgery worldwide magnifies the burden of this risk. Meticulous attention to watertight incision closure is critical in the prevention of endophthalmitis, particularly when clear corneal incisions are employed.

Finally, corneal sutures should be removed as soon as possible after wound integrity has been restored, and may be done at the one week post-operative visit. The will help ensure that that even an adequate suture does not break or become loose over time and act as a nidus of infection even years later.

While cataract surgery remains one of the most successful and safe procedures in ophthalmology, rare but serious infectious complications can arise. Proper preoperative preparation, sterile perioperative surgical technique, proper wound construction, postoperative removal of sutures, vigilance of early signs of infection, and aggressive early treatment of infection are keys to prevention of devastating post-surgical infections.

\section{Disclaimer}

The authors declare that they have no conflicts of interest or proprietary interests to disclose.

\section{References}

Results of the Endophthalmitis Vitrectomy Study. A randomized trial of immediate vitrectomy and of intravenous antibiotics for the treatment of postoperative bacterial endophthalmitis. Endophthalmitis Vitrectomy Study Group. Arch Ophthalmol, 1995. 113(12): p. 1479-96.

Prophylaxis of postoperative endophthalmitis following cataract surgery: results of the ESCRS multicenter study and identification of risk factors. J Cataract Refract Surg, 2007. 33(6): p. 978-88.

Al-Mezaine, H.S., A. Al-Assiri \&A.A. Al-Rajhi, Incidence, clinical features, causative organisms, and visual outcomes of delayed-onset pseudophakic endophthalmitis. Eur J Ophthalmol, 2009. 19(5): p. 804-11.

Aldave, A.J., J.D. Stein, V.A. Deramo, G.K. Shah, D.H. Fischer, et al., Treatment strategies for postoperative Propionibacterium acnes endophthalmitis. Ophthalmology, 1999. 106(12): p. 2395-401.

Ciulla, T.A., M.B. Starr \&S. Masket, Bacterial endophthalmitis prophylaxis for cataract surgery: an evidence-based update. Ophthalmology, 2002. 109(1): p. 13-24.

Clark, W.L., P.K. Kaiser, H.W. Flynn, Jr., A. Belfort, D. Miller, et al., Treatment strategies and visual acuity outcomes in chronic postoperative Propionibacterium acnes endophthalmitis. Ophthalmology, 1999. 106(9): p. 1665-70.

Dickey, J.B., K.D. Thompson \&W.M. Jay, Anterior chamber aspirate cultures after uncomplicated cataract surgery. Am J Ophthalmol, 1991. 112(3): p. 278-82.

Haapala, T.T., L. Nelimarkka, J.M. Saari, V. Ahola \&K.M. Saari, Endophthalmitis following cataract surgery in southwest Finland from 1987 to 2000. Graefes Arch Clin Exp Ophthalmol, 2005. 243(10): p. 1010-7. 
Holmberg, A., R. Lood, M. Morgelin, B. Soderquist, E. Holst, et al., Biofilm formation by Propionibacterium acnes is a characteristic of invasive isolates. Clin Microbiol Infect, 2009. 15(8): p. 787-95.

Kalpadakis, P., I. Tsinopoulos, G. Rudolph, K. Schebitz \&S.J. Froehlich, A comparison of endophthalmitis after phacoemulsification or extracapsular cataract extraction in a socio-economically deprived environment: a retrospective analysis of 2446 patients. Eur J Ophthalmol, 2002. 12(5): p. 395-400.

Kattan, H.M., H.W. Flynn, Jr., S.C. Pflugfelder, C. Robertson \&R.K. Forster, Nosocomial endophthalmitis survey. Current incidence of infection after intraocular surgery. Ophthalmology, 1991. 98(2): p. 227-38.

Kodjikian, L., A. Salvanet-Bouccara, S. Grillon, F. Forestier, J.L. Seegmuller, et al., Postcataract acute endophthalmitis in France: national prospective survey. J Cataract Refract Surg, 2009. 35(1): p. 89-97.

Leong, J.K., R. Shah, P.J. McCluskey, R.A. Benn \&R.F. Taylor, Bacterial contamination of the anterior chamber during phacoemulsification cataract surgery. J Cataract Refract Surg, 2002. 28(5): p. 826-33.

Lloyd, J.C. \&R. Braga-Mele, Incidence of postoperative endophthalmitis in a high-volume cataract surgicentre in Canada. Can J Ophthalmol, 2009. 44(3): p. 288-92.

Lundstrom, M., G. Wejde, U. Stenevi, W. Thorburn \&P. Montan, Endophthalmitis after cataract surgery: a nationwide prospective study evaluating incidence in relation to incision type and location. Ophthalmology, 2007. 114(5): p. 866-70.

Mollan, S.P., A. Gao, A. Lockwood, O.M. Durrani \&L. Butler, Postcataract endophthalmitis: incidence and microbial isolates in a United Kingdom region from 1996 through 2004. J Cataract Refract Surg, 2007. 33(2): p. 265-8.

Ormerod, L.D., J.E. Puklin, J.G. McHenry \&M.L. McDermott, Scleral flap necrosis and infectious endophthalmitis after cataract surgery with a scleral tunnel incision. Ophthalmology, 1993. 100(2): p. 159-63.

Ou, J.I. \&C.N. Ta, Endophthalmitis prophylaxis. Ophthalmol Clin North Am, 2006. 19(4): p. 449-56.

Ravindran, R.D., R. Venkatesh, D.F. Chang, S. Sengupta, J. Gyatsho, et al., Incidence of postcataract endophthalmitis at Aravind Eye Hospital: outcomes of more than 42,000 consecutive cases using standardized sterilization and prophylaxis protocols. J Cataract Refract Surg, 2009. 35(4): p. 629-36.

Sherwood, D.R., W.J. Rich, J.S. Jacob, R.J. Hart \&Y.L. Fairchild, Bacterial contamination of intraocular and extraocular fluids during extracapsular cataract extraction. Eye (Lond), 1989. 3 ( Pt 3): p. 308-12.

Speaker, M.G., F.A. Milch, M.K. Shah, W. Eisner \&B.N. Kreiswirth, Role of external bacterial flora in the pathogenesis of acute postoperative endophthalmitis. Ophthalmology, 1991. 98(5): p. 639-49; discussion 650.

Srinivasan, R., S. Tiroumal, R. Kanungo \&M.K. Natarajan, Microbial contamination of the anterior chamber during phacoemulsification. J Cataract Refract Surg, 2002. 28(12): p. 2173-6.

Taban, M., A. Behrens, R.L. Newcomb, M.Y. Nobe, G. Saedi, et al., Acute endophthalmitis following cataract surgery: a systematic review of the literature. Arch Ophthalmol, 2005. 123(5): p. 613-20.

Vafidis, G.C., R.J. Marsh \&A.R. Stacey, Bacterial contamination of intraocular lens surgery. Br J Ophthalmol, 1984. 68(8): p. 520-3. 